\title{
MicroRNA-9-5p functions as a tumor suppressor in papillary thyroid cancer via targeting BRAF
}

\author{
FENG GUO, XINMING HOU and QINGHUI SUN \\ Department of Thyroid and Breast Surgery, The Second People's Hospital of Liaocheng, \\ Liaocheng, Shandong 252600, P.R. China
}

Received January 18, 2016; Accepted July 5, 2018

DOI: $10.3892 / \mathrm{ol} .2018 .9423$

\begin{abstract}
MicroRNAs (miRNAs/miRs) are widely studied as key regulators of gene expression and are involved in various diseases by affecting the miRNA-mediated regulatory function. BRAF is an important oncogene in the regulation of cell proliferation and apoptosis. In the present study, reverse transcription-quantitative polymerase chain reaction was used to determine the expression levels of miR-9-5p and BRAF mRNA in patients with papillary thyroid cancer (PTC). Western blotting was used to detect BRAF protein level. A luciferase assay was used to verify the miR-9-5p target site in BRAF. Cell Counting Kit- 8 and flow cytometry were used to assess cell proliferation, and apoptosis, respectively. In the present study, it was demonstrated that miR-9-5p is downregulated in malignant PTC. Using bioinformatics analysis, miR-9-5p was predicted to target the human BRAF 3'-untranslated region (3'-UTR). A dual-luciferase assay demonstrated that miR-9-5p downregulated BRAF expression by directly targeting its 3'-UTR. Mutations in the 3'-UTR of BRAF completely abolished its interaction with miR-9-5p. Expression of exogenous miRNA that mimics miR-9-5p miRNA decreased BRAF protein and mRNA levels, while suppression of endogenous miR-9-5p resulted in an increase in BRAF protein, and mRNA levels. Furthermore, regulation of miR-9-5p was observed to suppress the viability of PTC cells by inducing apoptosis. Consistently, downregulation of miR-9-5p promoted proliferation of PTC cells by inhibiting the apoptosis of cells. In conclusion, the present study demonstrated that miR-9-5p may perform an important role in PTC prognosis and therapy.
\end{abstract}

Correspondence to: Dr Xinming Hou, Department of Thyroid and Breast Surgery, The Second People's Hospital of Liaocheng, 306 Jiankang Road, Liaocheng, Shandong 252600, P.R. China E-mail: thyroidca@163.com

Key words: microRNA-9-5p, tumor suppressor, papillary thyroid cancer, BRAF, apoptosis

\section{Introduction}

Globally, thyroid cancer is the eighth leading type of malignancy in women, accounting for $3 \%$ of all cancer types in women. The worldwide prevalence of thyroid cancer 15 years ago was $1.7 \%$, which ranked 14 th among all cancer types, and this value has now doubled. Each year, there are more incident cases of thyroid cancer compared with any other type of cancer (3.8\%/year between 1992 and 2001) (1). Currently, it is estimated that there are 5-8 thyroid cancer cases/105 individuals each year in developed countries (2-5). In addition, the mortality rate of males with thyroid cancer has risen faster compared with other types of cancer, following adjustment of age (2.3\% each year between 1992 and 2001), and papillary thyroid cancer (PTC) accounts for the majority of thyroid cancer types worldwide (5). In 2013, it was estimated that 60,000 cases would be newly diagnosed in the USA, and this number is increasing, which makes thyroid cancer an escalating and common disease (6,7). Although conventional options, including surgery and radioactive iodine are potent for PTC, they are not effective for undifferentiated (anaplastic) thyroid carcinoma (ATC), and advanced PTC, which are radioactive iodine-resistant (8).

A previous study reported an association between BRAF mutation, and the onset and development of PTC, as well as with the unfavorable prognosis of PTC (9). It has been demonstrated that mutations of the BRAF gene induce uncontrolled and persistent activation of kinase signaling pathways, causing over-proliferation, and differentiation into cancer cells (10). Notably, mutation of BRAF was identified in PTC progression of all stages. The presence of BRAF mutation in microcarcinoma or PTC at a very early stage indicates that this event may serve a role in the development of PTC tumorigenesis and susceptibility to another genetic variation, resulting in a more aggressive progression of PTC (9).

MicroRNAs (miRNAs) were identified at the turn of the 21st century, marking the milestone in cell biology of a new era. Changing the concept of the association between gene mRNAs and human disease has extended to contain those sequences in the residual $\sim 90 \%$ of eukaryotic genomes involving generation of non-coding RNAs. miRNAs, which as meta-controllers of gene expression, are essential for the cellular alterations required for development. It is important to have detailed knowledge of the mechanisms involved in the 
regulation of mRNA expression, particularly considering that dysregulation of miRNAs is associated with several thyroid disorders, including, medullary thyroid carcinoma, follicular thyroid carcinoma and thyroid adenoma (11).

It has been previously demonstrated that miR-9-5p is differentially-expressed in PTC (12), and dysregulation of BRAF has also been reported to be involved in the molecular mechanism of PTC cell apoptosis $(13,14)$. By searching the online miRNA database (www.targetscan.org), BRAF was revealed to be a virtual target of miR-9-5p with high similarity among species. In the present study, BRAF was validated as a target of miR-9-5p, and the involvement of miR-9-5p and BRAF in the development of PTC was verified.

\section{Materials and methods}

Sample collection. A total of 25 female patients (age, $45 \pm 0.38$ years; age range 44 years and 7 months- 45 years and 4 months old) who were diagnosed with PTC and underwent surgery were recruited for the present study between February 2014 and February 2015 at the Department of Thyroid and Breast Surgery, The Second People's Hospital of Liaocheng (Liaocheng, China). An additional 25 benign thyroid nodule tissue samples without PTC were selected as controls. Subsequent to selecting patients, diagnosis was confirmed by two independent pathologists blinded to the clinical results. The protocol of the present study was approved by the Ethics and Research Committees of The Second People's Hospital of Liaocheng, and written informed consent was obtained from each patient prior to their involvement in the study.

RNA isolation and reverse transcription-quantitative polymerase chain reaction $(R T-q P C R)$. The miRNeasy ${ }^{\circledR}$ Mini kit (TRIzol ${ }^{\circledR}$; Invitrogen; Thermo Fisher Scientific, Inc., Waltham, MA, USA) was used to extract the total RNA from BCPAP cells, obtained from the Cell Bank of Type Culture Collection of Chinese Academy of Sciences (Shanghai, China) in accordance with manufacturer's protocol. The RNeasy Mini spin column (Qiagen China Co., Ltd., Shanghai, China) was used to purify the target RNA according to the manufacturer's protocol, with a $\mathrm{P} 260 / 280 \mathrm{~nm}$ ratio $>1.8$ considered acceptable. For subsequent experiments, the RNA was stored at $-80^{\circ} \mathrm{C}$ in microcentrifuge tubes.

The ThermoScript RT-PCR system (Invitrogen; Thermo Fisher Scientific, Inc.) was used according to the manufacturer's protocol to reverse transcribe total RNA into cDNA. Fresh tissues were used to extract $1 \mathrm{mg}$ RNA for the synthesis of cDNA with a mixture containing $5 \mathrm{X}$ cDNA synthesis buffer (250 mM Tris acetate $\mathrm{pH} 8.4$ ), $40 \mathrm{nM}$ magnesium acetate and $375 \mathrm{mM}$ potassium acetate, and $10 \mathrm{mM}$ dNTP mix, $15 \mathrm{U}$ thermoscript transcriptase, $1 \mathrm{ml}$ random hexamers (50 ng/ml), $0.1 \mathrm{M}$ dithiothreitol and $40 \mathrm{U}$ RNaseOUT (Invitrogen; Thermo Fisher Scientific, Inc.) in a final volume of $20 \mathrm{ml}$. The sequences used were as follows: miR-9-5p forward, 5'-CGAGCTCTGTGTGTGTGTGTGTGTG-3'; reverse, 5'-TTCCGCGGCCGCTATGGCCGACGTCGACGG GAATGGGGAAAGGGAA-3'; BRAF forward, 5'-GGCGGC AGCGCTGTGGCGGCG-3'; and reverse, 5'-CGTAGGGTC ATACTCATCCAC-3'. The reaction was performed at $18^{\circ} \mathrm{C}$ for $25 \mathrm{~min}, 42^{\circ} \mathrm{C}$ for $30 \mathrm{~min}$ and $90^{\circ} \mathrm{C}$ for $7 \mathrm{~min}$.
For qPCR, a SYBR-Green fluorophore (Applied Biosystems, Foster City, CA, USA) was used, and the thermocycling protocol was $10 \mathrm{~min}$ at $95^{\circ} \mathrm{C}$, followed by 40 cycles of $30 \mathrm{sec}$ at $95^{\circ} \mathrm{C}, 30 \mathrm{sec}$ at $60^{\circ} \mathrm{C}$ and $30 \mathrm{sec}$ at $72^{\circ} \mathrm{C}$. The expression of BRAF mRNA and miR-9-5p was calculated relative to the expression level of the endogenous control, $\beta$-actin. The $2^{-\Delta \Delta \mathrm{Cq}}$ method (15) was used to analyze the relative quantification of BRAF mRNA. Expression of miR-9-5p was normalized to U6, the sequence of primer for U6 is: Forward 5'-CTCGCTTCG GCAGCACA-3' and Reverse 5'-AACGCTTCACGAATTTGC GT-3'. Every experiment was performed at least three times.

Cell culture and transfection. Dulbecco's modified Eagle's medium (DMEM; Invitrogen; Thermo Fisher Scientific, Inc.), supplemented with $50 \mathrm{mg} / \mathrm{ml}$ streptomycin (Invitrogen; Thermo Fisher Scientific, Inc.), $10 \%$ fetal bovine serum (FBS; Atlanta Biologicals, Lawrenceville, GA, USA) and $50 \mathrm{mg} / \mathrm{ml}$ penicillin was used to maintain BCPAP cells in an atmosphere of $5 \% \mathrm{CO}_{2}$ and $95 \%$ air at $37^{\circ} \mathrm{C}$. The BCPAP cells were seeded on 6-well plates containing growth medium and antibiotics at a density of $3-6 \times 10^{5}$ cells/well for $12 \mathrm{~h}$ prior to transfection. When $80 \%$ confluence was achieved, $100 \mathrm{pM}$ miR-9-5p mimics; CGAGCTCTGTGTGTGTGTGTGTGT G-3; miR-9-5p inhibitors; 5'-TTCCGCGGCCGCTATGGC CGACGTCGACGGGAATGGGGAAAGGGAA-3', or BRAF small interfering RNA; 5'-GGCGCCTCCCTTCCCCCTCCC CTT-3' (siRNA; all Invitrogen; Thermo Fisher Scientific, Inc.) were transfected into the cells using $2 \mu \mathrm{l}$ Lipofectamine 2000 (Invitrogen; Thermo Fisher Scientific, Inc.) for $30 \mathrm{~min}$ at $37^{\circ} \mathrm{C}$. The time interval between the completion of transfection and the use of the transfected cells was $4 \mathrm{~h}$. The sequence of siRNA was: 5'-AAGUGGCAUGGUGAUGUGGCA-3', and the final concentration used for transfection was 20 pmol.

Vector construction and mutagenesis. In order to determine miRNA targeting of the 3'-UTR region of BRAF, the full length of the BRAF 3'-UTR was amplified and cloned into a pmiR-REPORT luciferase vector (Promega Corporation, Madison, WI, USA). DNA was extracted from the tissue samples using the PicoPure ${ }^{\mathrm{TM}}$ DNA Extraction Kit (Applied Biosystems; Thermo Fisher Scientific, Inc.), and treated with DNA polymerase (Promega Corporation). The sequences of the primers were as follows: BRAF forward, 5'-GGCGGC AGCGCTGTGGCGGCG-3'; and reverse, 5'-CGTAGGGTC ATACTCATCCAC-3'. The temperature protocol was as follows: $95^{\circ} \mathrm{C}$ for $3 \mathrm{~min}$, followed by 30 cycles of $94^{\circ} \mathrm{C}$ for $40 \mathrm{sec}, 56^{\circ} \mathrm{C}$ for $35 \mathrm{sec}$ and final extension at $72^{\circ} \mathrm{C}$ for $60 \mathrm{sec}$. The Vybrant ${ }^{\mathrm{TM}}$ MTT Cell Proliferation Assay kit (Thermo Fisher Scientific, Inc.) was used to generate the mutated vector by replacing the miR-9-5p binding site nucleotides, according to the manufacturer's protocol.

Cell proliferation assay. An MTT assay was performed to analyze the proliferation of BCPAP cells in accordance with the manufacturer's protocol (Thermo Fisher Scientific, Inc.). DMEM supplemented with $10 \%$ FBS was used to incubate the BCPAP cells on 96-well plates at a final concentration of $3 \times 10^{4}$ cells/ml. MTT (10 $\mu \mathrm{l}$; Thermo Fisher Scientific, Inc.) was then added to each well, and the culture medium was incubated for 1,2 and 3 days at $37^{\circ} \mathrm{C}$. Subsequent to culturing 
for an additional $48 \mathrm{~h}, 1 \%$ dimethyl sulfoxide was used to dissolve the formazan crystals, a blood counting chamber was used to count the number of cells, and an ELISA plate reader was used to analyze the proliferation of BCPAP cells based on the absorbance, which was read at $440 \mathrm{~nm}$.

Luciferase assay. The luciferase reaction mixture included $1 \times 10^{6}$ BCPAP cells, $1 \mu \mathrm{g}$ of a Renilla luciferase expression construct pRL-TK (Promega Corporation), $1 \mu \mathrm{g}$ p-BRAF (wild-type or mutant) plasmid and 50 pmol miR-9-5p mimics (or control). The dual-luciferase assay system (Promega Corporation) was used to detect the luciferase activity according to Renilla luciferase activity at $36 \mathrm{~h}$ post-transfection, according to the manufacturer's protocol. Each experiment was performed at least three times, and transfection was performed with Lipofectamine $2000^{\circledR}$ (Invitrogen; Thermo Fisher Scientific, Inc.).

Western blot analysis. For analysis of the expression of BRAF mRNA and miR-9-5p from tissue samples and BCPAP cells, radio immunoprecipitation assay lysis buffer (Sigma-Aldrich; Merck KGaA, Darmstadt, Germany) containing protease inhibitors (Roche Applied Science, Madison, WI, USA), 5 g/l sodium deoxycholate, $0.2 \mathrm{~g} / 1 \mathrm{NaN} 3,10 \mathrm{ml} / 1 \mathrm{NP}-40,150 \mathrm{mmol} / 1$ $\mathrm{NaCl} 100 \mu \mathrm{g} / \mathrm{ml}, 0.1 \mathrm{~g} / \mathrm{l} \mathrm{SDS}$, phenylmethylsulfonyl fluoride, $1 \mu \mathrm{g} / \mathrm{ml}$ aprotinin and $50 \mathrm{mmol} / 1$ Tris- $\mathrm{HCl}(\mathrm{pH} 8.5)$ was used to lyse the BCPAP cells following transfection, according to manufacturer's protocol, followed by two washes with PBS (Invitrogen; Thermo Fisher Scientific, Inc.). Protein determination was performed using a BCA assay (Beyotime Institute of Biotechnology, Haimen, China). SDS-PAGE (12\%) was used to purify the target proteins, and proteins ( $35 \mu \mathrm{g} / \mathrm{lane})$ were then transferred to a polyvinylidene fluoride membrane (GE Healthcare, Chicago, IL, USA). Tween-20 or TBS with $5 \%$ non-fat milk (Sigma-Aldrich; Merck KGaA) was used to block the membrane for $2 \mathrm{~h}$ at room temperature. The primary anti-BRAF antibodies in TBS buffer (dilution, 1:1,000; cat. no., sc-136263; Santa Cruz Biotechnology, Inc., Dallas, TX, USA) were used to detect the target protein via incubation for $12 \mathrm{~h}$ at $4^{\circ} \mathrm{C}$. Subsequently, membranes were washed twice with PBS, followed by incubation with horseradish peroxidase-conjugated secondary antibody (dilution, 1:3,000; cat.no 7075S Cell Signaling Technologies, Inc., Danvers, $\mathrm{MA}$, USA) for another $2 \mathrm{~h}$ at $25^{\circ} \mathrm{C}$. Prior to film exposure, a peroxidase substrate was used to enhance chemiluminescence (Thermo Fisher Scientific, Inc.). Amersham ECL Prime Western Blotting Detection reagent (cat. no. RPN2236; GE Healthcare, Chicago, IL, USA) was used to achieve visualization of the relative enrichment of proteins. Bio-Rad Chemi-DocXRS (Bio-Rad Laboratories, Hercules, CA, USA) was utilized to visualize band of target protein.

Apoptosis analysis. In order to analyze apoptosis, 7-amino-actinomycin D (7-AAD) was used to dual stain the BCPAP cells after transfecting for $72 \mathrm{~h}$, and the Annexin V-fluorescein isothiocyanate (FITC)/7-AAD kit (Beckman Coulter, Inc., Brea, CA, USA) was used to detect the Annexin V-FITC, according to the manufacturer's protocol. Flow cytometry (Cell Lab Quanta SC; Beckman Coulter, Inc.) with 585/42 nm (PI) and 530/30 nm (FITC) emission filters was used to analyze the apoptosis of cells after staining cells according to the manufacturer's protocol. FCAP Array Software v3.0 was used for analysis (Cell Lab Quanta SC; Beckman Coulter, Inc.). All experiments were performed in triplicate.

Statistical analysis. All values are presented as the mean \pm standard deviation. For the bioinformatics analysis, computational algorithms (www.targetscan.org; access date 02/02/2015) were used to predict putative miR-9-5p targets. Fisher's exact test was used to analyze the comparisons of categorical variables. Comparisons among the continuous variables were performed using Spearman's Rank Correlation and Sign test. $\mathrm{P}<0.05$ was considered to indicate a statistically significant difference. StatView Software (v.5, SAS Institute, Inc., Cary, NC, USA) was used to analyze the statistical analysis.

\section{Results}

miR-9-5p is downregulated in malignant PTC. To identify miRNAs regulating malignant PTC, miRNA expression levels were analyzed between PTC and benign thyroid nodules. miR-9-5p was consistently downregulated in malignant PTC compared with benign tumor samples $(\mathrm{P}<0.05$ vs. Thyroid nodule tissue samples; Fig. 1). Therefore, the present study focused on the function of miR-9-5p, which was hypothesized to distinguish the malignant tumors from the benign ones.

BRAF expression transcripts are targeted by miR-9-5p. One of the biggest challenges in studying miRNAs is to identify target genes and associate their downregulation with cellular properties. Computational algorithms (www.targetscan.org) have been used to predict putative miR-9-5p targets based on complementarity to the 3'-UTR of the target mRNA. Through bioinformatics analysis, miR-9-5p was predicted to target the human BRAF 3'-UTR (Fig. 2). To investigate whether miR-9-5p is a real BRAF regulator, the following experiments were performed. Firstly, luciferase reporter vectors containing a wild-type or mutation BRAF 3'-UTR sequence at the downstream of luciferase were constructed (Fig. 2). miR-9-5p mimics and corresponding reporter vectors were co-transfected into the BCPAP cell line. A dual-luciferase assay was performed at $48 \mathrm{~h}$ post-transfection. The results of the dual-luciferase assay revealed that when the reporter vector contained a wild-type BRAF 3'-UTR, miR-9-5p significantly suppressed the luciferase expression compared with the scramble control group $(\mathrm{P}<0.05$ vs. scramble control) (Fig. 3). Consistently, mutation of the target region completely abolished this observation, returning levels of luciferase activity similar to that of the scramble control group (Fig. 3).

BRAF expression is upregulated in PTC. It was hypothesized that the reduction of miR-9-5p leads to the increase of BRAF expression level in malignant papillary thyroid tumors. To support this hypothesis, RT-qPCR and western blot analysis was used to determine the expression level of BRAF mRNA, and protein in the two groups (Fig. 4). The results demonstrated that BRAF mRNA and protein expression was 


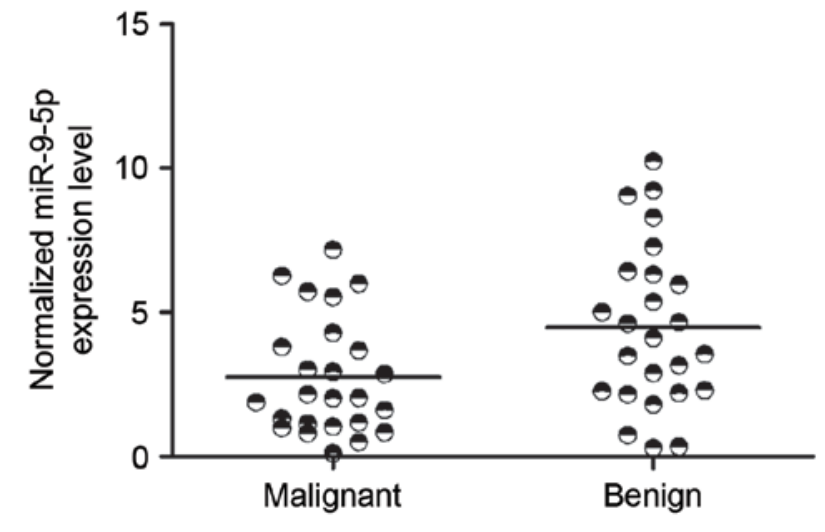

Figure 1. miR-9-5p is downregulated in malignant PTC. Expression levels of miR-9-5p were detected in RNA samples extracted from malignant $(n=25)$ or benign $(n=25)$ PTC tissues. Expression of miR-9-5p was normalized to U6. miR-9-5p, microRNA-9-5p; PTC, papillary thyroid cancer.

$\begin{array}{ll}\text { hsa-miR-9-5p } & \text { 5'-UUUUUUUUAAGGUGAACCAAAGA-3' } \\ \text { Wild-type BRAF } & \text { 3'-AGUAUGUCGAUCUAUUGGUUUCU-5' } \\ \text { hsa-miR-9-5p } & \text { 5'-UUUUUUUUAAGGUGAACCAAAGA-3' } \\ \text { Mutant BRAF } & \text { 3'-AGUAUGUCGAUCUAUGUUGCGUU-5' }\end{array}$

Figure 2. Schematic illustrating the predicted miR-9-5p binding sites in the 3'-untranslated region of BRAF mRNA. BRAF mutations were constructed by destroying the base pairing between miRNA and its target in seed regions. miR-9-5p, microRNA-9-5p.

upregulated in malignant papillary thyroid tumor compared with that in benign samples $(\mathrm{P}<0.05$ vs. benign tumor samples).

Effect of miR-9-5p upregulation or downregulation on the expression of BRAF. To determine whether miR-9-5p disrupts BRAF expression in BCPAP cells, miR-9-5p mimics were transfected into BCPAP cells. Scramble served as a negative control, while BRAF siRNA served as a positive control. The data revealed that BRAF mRNA and protein level could be suppressed by miR-9-5p mimic ( $\mathrm{P}<0.05$ vs. scramble control) (Fig. 5). Furthermore, similar experiments were performed using miR-9-5p inhibitor treatment and the decrease in miR-9-5p increased BRAF mRNA and protein levels $(\mathrm{P}<0.05$ vs. scramble control) (Fig. 5). These results demonstrated that miR-9-5p targets BRAF 3'-UTR in a direct manner, therefore suppressing the expression of BRAF.

MiR-9-5p affects cell proliferation and apoptosis by regulating $B R A F$ expression. BRAF performs an important role in regulating cell proliferation, and high levels of BRAF may promote proliferation of malignant cells by suppressing apoptosis (16). To verify this, miR-9-5p mimics and inhibitor were introduced into PTC cells, with scramble serving as a negative control. As presented in Fig. 6, miR-9-5p and BRAF siRNA suppressed the viability of PTC cells by inducing apoptosis $(\mathrm{P}<0.05$ vs. scramble control), and consistently, downregulation of miR-9-5p promoted proliferation of PTC cells by inhibiting cell apoptosis $(\mathrm{P}<0.05$ vs. scramble control).

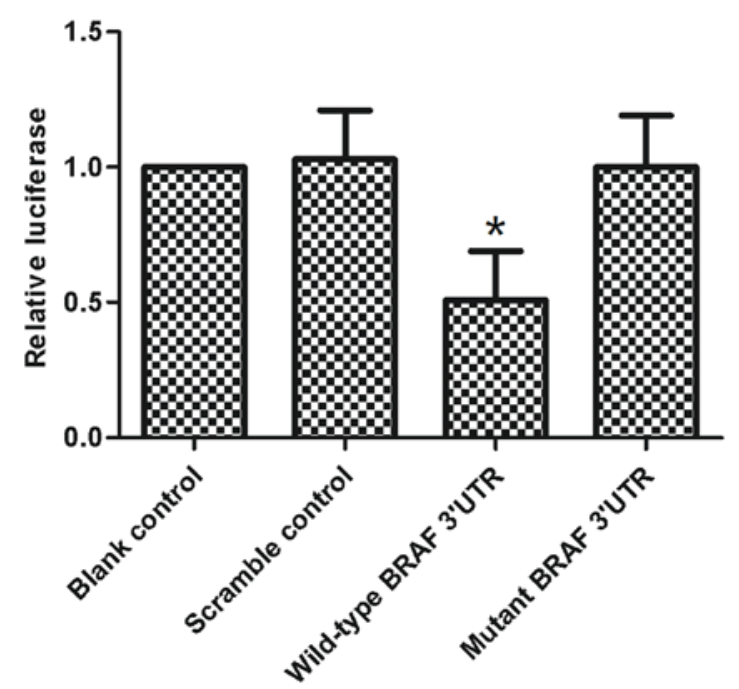

Figure 3. Luciferase activity of reporter genes containing a wild BRAF and mutant 3'-UTRs. PTC cells were co-transfected with miR-9-5p mimic and indicated BRAF 3'-UTR-luciferase reporter. Blank and scramble served as negative controls. Luciferase activity was assayed $48 \mathrm{~h}$ later. All data are presented as the mean \pm standard deviation, normalized to the blank control of three independent experiments. miR-9-5p, microRNA-9-5p; 3'-UTR, 3'-untranslated region. " $\mathrm{P}<0.05$ compared with the blank controls.
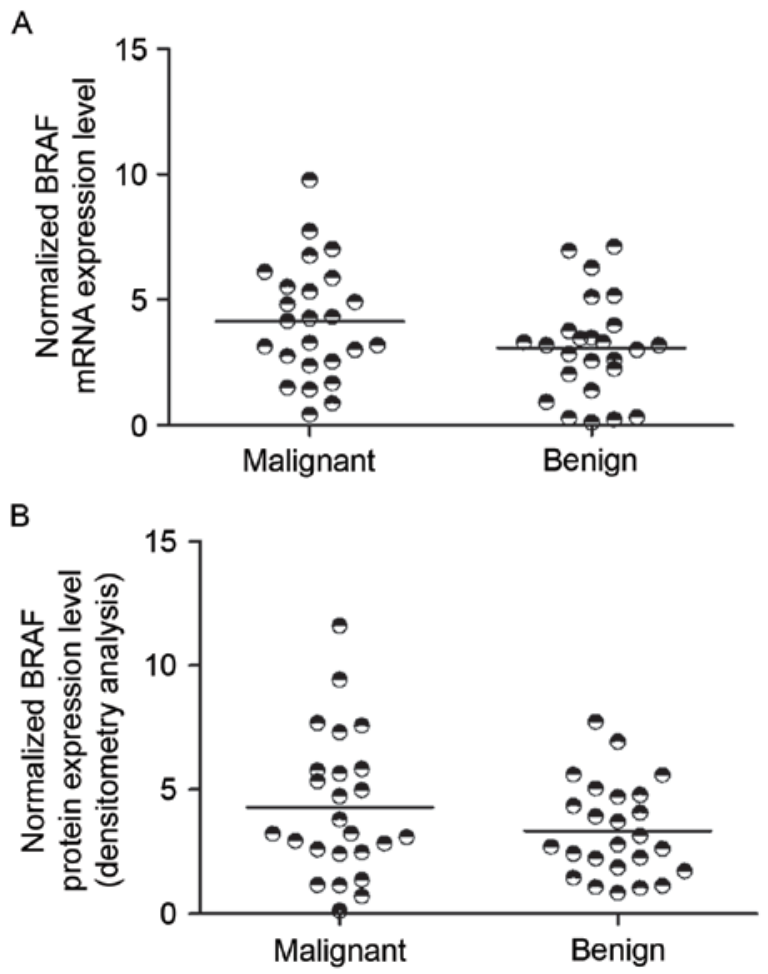

Figure 4. BRAF mRNA and protein levels were upregulated in malignant papillary thyroid tumor. The expression levels of (A) BRAF mRNA and (B) BRAF protein were compared between malignant $(n=25)$, and benign $(\mathrm{n}=25)$ papillary thyroid tumors.

\section{Discussion}

MiRNAs have been reported to be functionally involved in the regulation of biological processes, and may function as oncogenes or tumor suppressors in the development of 


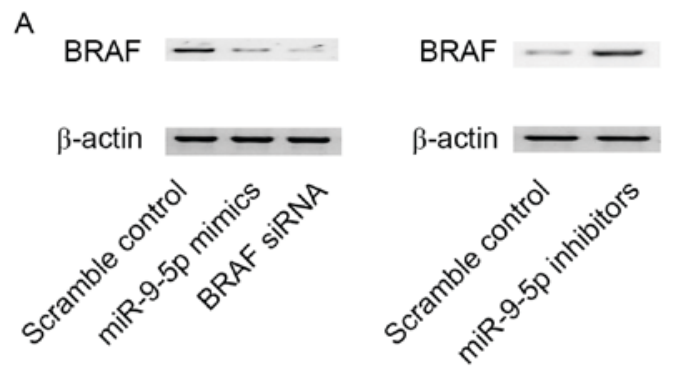

B

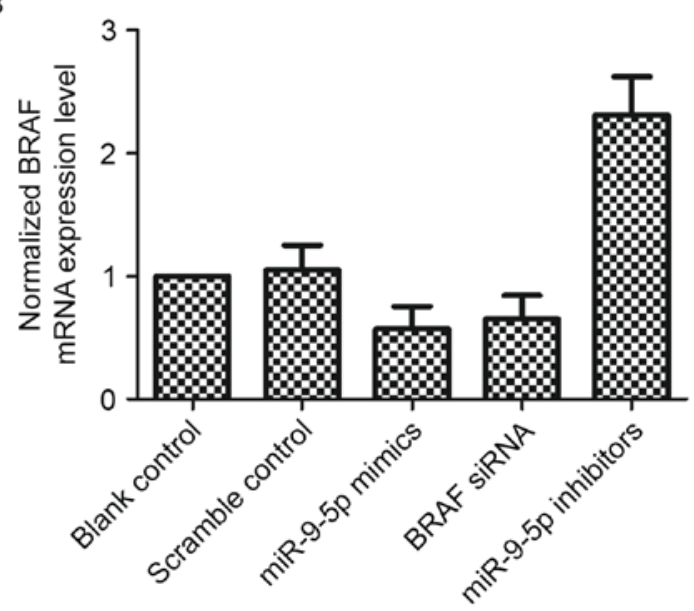

Figure 5. BRAF protein level and mRNA level can be inhibited by miR-9-5p. miR-9-5p mimics or inhibitor were transfected into BCPAP cells. (A) Western blot analysis was performed to analyze BRAF protein level. (B) Relative expression levels of BRAF mRNA were determined by reverse transcription-quantitative polymerase chain reaction. Blank and scramble controls served as negative controls. BRAF siRNA served as a positive control. Values are presented as the mean \pm standard deviation. miR-9-5p, microRNA-9-5p; siRNA, small interfering RNA.

various malignancies (10). The invasiveness of mesothelial cells (MCs) is markedly reduced when the levels of miR-9-5p are increased, which reveals a mechanism involving prevention of build-up of myofibroblasts derived from MCs in the submesothelial compact zone of the peritoneum, thereby preventing it from initiating the process of fibrosis (17). Notably, over-expression of miR-9-5p also reduced NADPH oxidase 4 levels induced by transforming growth factor- $\beta$ (TGF- $\beta$ ) 1 and TGF- $\beta$ receptor 2 , representing a possible mechanism involving the anti-fibrotic effect of miR-9-5p in pleural mesothelial cells (18). The level of miR-9-5p in human non-epithelial cells was revealed to be higher compared with that in epithelial MCs, which demonstrates that miR-9-5p protects against the development of pulmonary fibrosis (19). In the present study, miRNA expression levels were analyzed between PTC and benign thyroid nodule tissue samples. MiR-9-5p was consistently downregulated in PTC compared with the normal control.

To identify the downstream effector of miR-9-5p, bioinformatics analysis was performed and revealed that miR-9-5p was predicted to target the human BRAF 3'-UTR. Furthermore, a luciferase assay was performed, demonstrating that when the reporter vector contained a wild-type BRAF 3'-UTR, miR-9-5p significantly suppressed the luciferase expression. Consistent with this, mutation of target regions completely abolished this interaction. In addition, miR-9-5p mimics were

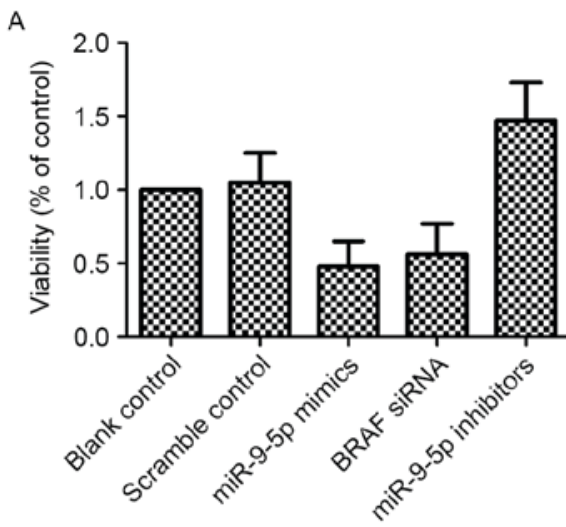

B

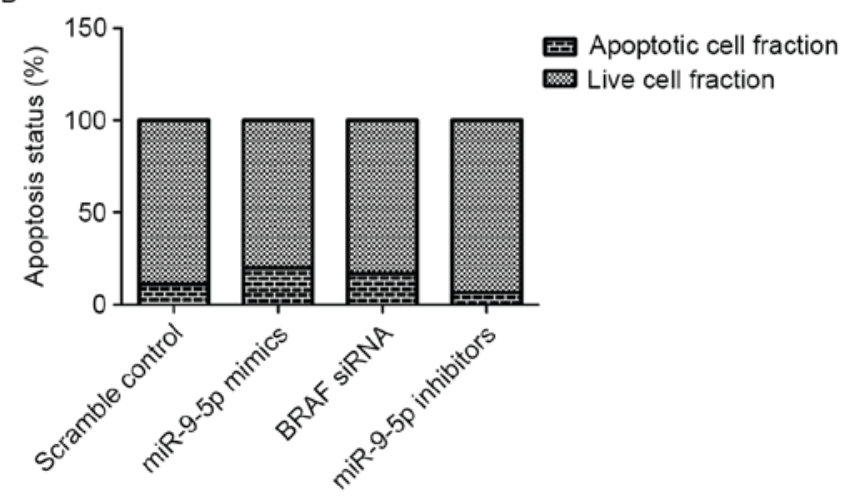

Figure 6. Effect of miR-9-5p on viability and apoptosis in papillary thyroid cells. (A) miR-9-5p mimics or inhibitors were transfected into papillary thyroid cells. The Cell Counting Kit-8 assay was performed to determine the effect of miR-9-5p on papillary thyroid cell proliferation. Blank or scramble served as negative controls. (A) siRNA against BRAF served as a positive control. (B) miR-9-5p mimics or inhibitors were transfected into papillary thyroid cells. An apoptosis assay was performed to determine the effect of miR-9-5p on cell apoptosis. Scramble served as negative control. siRNA against BRAF served as a positive control. miR-9-5p, microRNA-9-5p; siRNA, small interfering RNA.

transfected into BCPAP cells, and scramble served as a negative control, while BRAF siRNA served as a positive control. The data demonstrated that BRAF mRNA and protein levels were be suppressed by miR-9-5p mimic. Furthermore, similar experiments were performed using miR-9-5p inhibitor treatment, and the decrease of miR-9-5p increased BRAF mRNA and protein levels. These results confirmed that miR-9-5p targets the BRAF 3'-UTR directly, and therefore suppresses the expression of BRAF.

The cascade of RAF-mitogen-activated protein kinase kinase (MEK)-extracellular signal-regulated kinase (ERK) protein kinases serves a major role in transforming extracellular mitogenic signals to cell proliferation, as well as other cellular functions $(20,21)$. The family of RAF Ser/Thr kinases includes A-, B- and C-RAF, which have similar domain structures. It has been demonstrated that hetero- and homo-dimerization of RAF family proteins causes an essential change in MEK phosphorylation and activation (kinase regulated by extracellular signal/protein kinase activated by mitogen), and consequently ERK (kinase regulated by extracellular signal) responding to the activation of RAS (22). Kinase suppressors of RAS proteins primarily serve as scaffolds of the signaling cascade and are also homologs of RAF family members, tethering 
ERKs, MEKs and RAFs together. In human cancer, the mutations are commonly present in the RAF-MEK-ERK signaling pathway (23). A total of $\sim 6 \%$ of all human cancers and $50 \%$ of melanomas exhibit BRAF mutations (24). In $>90 \%$ of BRAF mutations, there is a single base substitution present in a codon of the kinase domain, contributing to V600E amino acid alteration, constitutive active BRAF protein kinase, and subsequently downstream signaling of MEK-ERK (21). In the present study, it was hypothesized that the reduction of miR-9-5p leads to the increase of BRAF expression level in malignant papillary thyroid tumors. To test this hypothesis, RT-qPCR was used to determine the expression level of BRAF mRNA in two groups, and it was revealed that BRAF expression is upregulated and miR-9-5p is downregulated in malignant papillary thyroid tumor.

A transversion point mutation $(1799 \mathrm{~T}>\mathrm{A})$ in the BRAF gene has been observed in 20-40\% of ATCs and $45 \%$ of PTCs, leading to a valine to glutamate substitution at amino acid 600 of the protein termed BRAFV600E, and finally constitutive activation of kinase activity $(25,26)$. It is evident that BRAF mutants in tumors are not always a signal of clinically aggressive thyroid cancer in patients, although it is true that BRAFV600E has an important role in tumor behavior $(27,28)$. Other immune factors and gene pathways are either known or putatively unknown to interact with mutant BRAF signaling, which involves in the formation of aggressive features in thyroid tumors (29). Mutations that affect the phosphoinositide 3-kinase (PI3K)-protein kinase B (AKT) pathway and tumor suppressor p53 are among other genetic events revealed to activate dedifferentiation, and tumor progression (27). The majority of ATCs exhibit inactive p53 (30). The inactivation of the tumor suppressor phosphatase and tensin homolog is less prevalent compared with mutations of $\mathrm{p} 53$, which are observed in $\sim 15 \%$ of cases of ATC and contributes to the activation of the PI3K-AKT signaling pathway (31). Furthermore, efforts will be made to identify other putative relevant drivers of mutations and signaling pathways, including those made by The Cancer Genome Atlas (http://tcga-data.nci.nih.gov/tcga/). Thyroid cancer progression can be well studied using mouse models. Using orthotopic and genetically engineered approaches that have been designed more recently, the mouse models of PTC, and the more aggressive and lethal types of thyroid cancer, ATC, have been established (32). There are strengths and drawbacks to each of these approaches. A previous study identified an association between mortality rate and BRAF status, previously regarded as additional information to traditional factors of prognosis (33). The BRAF mutation may also be a potential marker for predicting the response to targeted treatments considering the availability of existing targeted treatments, including a BRAF inhibitor (34). However, there remains debate about the role of the BRAF mutation in prognosis, since a prognostic role for the BRAF mutation has not been identified in numerous studies $(35,36)$. In the present study, regulation of miR-9-5p was found to suppress the viability of PTC cells by inducing apoptosis, and consistently, downregulation of miR-9-5p promoted proliferation of PTC cells by inhibiting apoptosis of the cells.

In conclusion, the findings of the present study revealed that BRAF is a direct target of miR-9-5p, and the miR-9-5p/BRAF signaling pathway may perform an important role in the tumorigenesis of PTC. Furthermore, miR-9-5p/BRAF may be a therapeutic target in the treatment of PTC.

\section{Acknowledgements}

Not applicable.

\section{Funding}

No funding was received.

\section{Availability of data and materials}

The datasets generated and analyzed in the present study are included in this published article.

\section{Authors' contributions}

FG was responsible for the planning of the present study, data collection, analysis and interpretation and preparation of the manuscript. XH also participated in data collection, analysis and interpretation and was responsible for the collection of funds. QS was responsible for data analysis and interpretation, preparation of the manuscript and literature analysis.

\section{Ethics and consent to participate}

The protocol of the present study was approved by the Ethics and Research Committees of The Second People's Hospital of Liaocheng, and written informed consent was obtained from each patient prior to their involvement in the study.

\section{Patient consent for publication}

The study participants provided consent for the data in the present study to be published.

\section{Competing interests}

The authors declare that they have no competing interests.

\section{References}

1. Al-Brahim N and Asa SL: Papillary thyroid carcinoma: An overview. Arch Pathol Lab Med 130: 1057-1062, 2006.

2. Gomez Segovia I, Gallowitsch HJ, Kresnik E, Kumnig G, Igerc I, Matschnig S, Stronegger WJ and Lind P: Descriptive epidemiology of thyroid carcinoma in Carinthia, Austria: 1984-2001. Histopathologic features and tumor classification of 734 cases under elevated general iodination of table salt since 1990: Population-based age-stratified analysis on thyroid carcinoma incidence. Thyroid 14: 277-286, 2004.

3. Akslen LA, Haldorsen T, Thoresen SO and Glattre E: Incidence pattern of thyroid cancer in Norway: Influence of birth cohort and time period. Int J Cancer 53: 183-187, 1993.

4. Colonna M, Grosclaude P, Remontet L, Schvartz C Mace-Lesech J, Velten M, Guizard A, Tretarre B, et al: Incidence of thyroid cancer in adults recorded by French cancer registries (1978-1997). Eur J Cancer 38: 1762-1768, 2002.

5. Howe HL, Wingo PA, Thun MJ, Ries LA, Rosenberg HM, Feigal EG and Edwards BK: Annual report to the nation on the status of cancer (1973 through 1998), featuring cancers with recent increasing trends. J Natl Cancer Inst 93: 824-842, 2001.

6. Davies L and Welch HG: Increasing incidence of thyroid cancer in the United States, 1973-2002. JAMA 295: 2164-2167, 2006. 
7. Churilla TM, Donnelly PE, Leatherman ER, Adonizio CS and Peters CA: Total mastectomy or breast conservation therapy? how radiation oncologist accessibility determines treatment choice and quality: A SEER Data-base analysis. Breast J 21: 473-480, 2015

8. Schechter RB, Nagilla M, Joseph L, Reddy P, Khattri A, Watson S, Locati LD, Licitra L, Greco A, Pelosi G, et al: Genetic profiling of advanced radioactive iodine-resistant differentiated thyroid cancer and correlation with axitinib efficacy. Cancer Lett 359: 269-274, 2015.

9. Knauf JA, Ma X, Smith EP, Zhang L, Mitsutake N, Liao XH, Refetoff S, Nikiforov YE and Fagin JA: Targeted expression of BRAFV600E in thyroid cells of transgenic mice results in PTCs that undergo dedifferentiation. Cancer Res 65: 4238-4245, 2005.

10. Kundu A, Quirit JG, Khouri MG and Firestone GL: Inhibition of oncogenic BRAF activity by indole-3-carbinol disrupts microphthalmia-associated transcription factor expression and arrests melanoma cell proliferation. Mol Carcinog 56: 49-61, 2017.

11. Saugstad JA: MicroRNAs as effectors of brain function with roles in ischemia and injury, neuroprotection, and neurodegeneration. J Cereb Blood Flow Metab 30: 1564-1576, 2010.

12. Nalysnyk L, Cid-Ruzafa J, Rotella P and Esser D: Incidence and prevalence of idiopathic pulmonary fibrosis: Review of the literature. Eur Respir Rev 21: 355-361, 2012.

13. Sondermann A, Andreghetto FM, Moulatlet AC, da Silva Victor E, de Castro MG, Nunes FD, Brandão LG and Severino P. MiR-9 and miR-21 as prognostic biomarkers for recurrence in PTC. Clin Exp Metastasis 32: 521-530, 2015.

14. Yoon S, An YS, Lee SJ, So EY, Kim JH, Chung YS and Yoon JK: Relation between F-18 FDG uptake of PET/CT and BRAFV600E mutation in PTC. Medicine (Baltimore) 94: e2063, 2015.

15. Livak KJ and Schmittgen TD: Analysis of relative gene expression data using real-time quantitative PCR and the 2(-Delta Delta C(T)) method. Methods 25: 402-8, 2001.

16. Shen CH, Yuan P, Perez-Lorenzo R, Zhang Y, Lee SX, Ou Y Asara JM, Cantley LC and Zheng B: Phosphorylation of BRAF by AMPK impairs BRAF-KSR1 association and cell proliferation. Mol Cell 52: 161-172, 2013.

17. Rothenberg SM, Daniels GH and Wirth LJ: Redifferentiation of iodine-refractory BRAF V600E-Mutant metastatic papillary thyroid cancer with dabrafenib-response. Clin Cancer Res 21 : 5640-5641, 2015

18. Noble PW, Barkauskas CE and iang D: Pulmonary fibrosis: Patterns and perpetrators. J Clin Invest 122: 2756-2762, 2012.

19. Amara N, Goven D, Prost F, Muloway R, Crestani B and Boczkowski J: NOX4/NADPH oxidase expression is increased in pulmonary fibroblasts from patients with idiopathic pulmonary fibrosis and mediates TGFbeta1-induced fibroblast differentiation into myofibroblasts. Thorax 65: 733-738, 2010.

20. Udell CM, Rajakulendran T, Sicheri F and Therrien M: Mechanistic principles of RAF kinase signaling. Cell Mol Life Sci 68: 553-565, 2011.

21. Osborne JK, Zaganjor E and Cobb MH: Signal control through Raf: In sickness and in health. Cell Res 22: 14-22, 2012

22. Roberts PJ and Der V: Targeting the Raf-MEK-ERK mitogen-activated protein kinase cascade for the treatment of cancer. Oncogene 26: 3291-3310, 2007.
23. Matallanas D, Birtwistle M, Romano D, Zebisch A, Rauch J, von Kriegsheim A and Kolch W: Raf Family Kinases: Old dogs have learned new tricks. Genes Cancer 2: 232-260, 2011.

24. Gray-Schopfer V, Wellbrock C and Marais R: Melanoma biology and new targeted therapy. Nature 445: 851-857, 2007.

25. American Thyroid Association (ATA) Guidelines Taskforce on Thyroid Nodules and Differentiated Thyroid Cancer, Cooper DS, Doherty GM, Haugen BR, Kloos RT, Lee SL, Mandel SJ, Mazzaferri EL, McIver B, Pacini F, et al: Revised American Thyroid Association management guidelines for patients with thyroid nodules and differentiated thyroid cancer. Thyroid 19: 1167-1214, 2009.

26. Tuttle RM, Ball DW, Byrd D, Dilawari RA, Doherty GM, Duh QY, Ehya H, Farrar WB, Haddad RI, Kandeel F, et al: Thyroid carcinoma. J Natl Compr Canc Netw 8: 1228-1274, 2010.

27. Brown RL, de Souza JA and Cohen EE: Thyroid cancer: Burden of illness and management of disease. J Cancer 2: 193-199, 2011.

28. Cohen Y, Xing M, Mambo E, Guo Z, Wu G, Trink B, Beller U, Westra WH, Ladenson PW and Sidransky D: BRAF mutation in papillary thyroid carcinoma. J Natl Cancer Inst 95: 625-627, 2003.

29. Lin KL, Wang OC, Zhang XH, Dai XX, Hu XQ and Qu JM: The BRAF mutation is predictive of aggressive clinicopathological characteristics in papillary thyroid microcarcinoma. Ann Surg Oncol 1: 3294-3300, 2010.

30. Kimura ET, Nikiforova MN, Zhu Z, Knauf JA, Nikiforov YE and Fagin JA: High prevalence of BRAF mutations in thyroid cancer: Genetic evidence for constitutive activation of the RET/PTC-RAS-BRAF signaling pathway in papillary thyroid carcinoma. Cancer Res 63: 1454-1457, 2003.

31. Soares P, Trovisco V, Rocha AS, Lima J, Castro P, Preto A, Máximo V, Botelho T, Seruca R and Sobrinho-Simões M: BRAF mutations and RET/PTC rearrangements are alternative events in the etiopathogenesis of PTC. Oncogene 22: 4578-4580, 2003.

32. Vanden Borre P, McFadden DG, Gunda V, Sadow PM, Varmeh S, Bernasconi M, Jacks T and Parangi S: The next generation of orthotopic thyroid cancer models: immunocompetent orthotopic mouse models of BRAF V600E-positive papillary and anaplastic thyroid carcinoma. Thyroid 24: 705-714, 2014.

33. Xing M, Alzahrani AS, Carson KA, Viola D, Elisei R, Bendlova B, Yip L, Mian C, Vianello F, Tuttle RM, et al: Association between BRAF V600E mutation and mortality in patients with papillary thyroid cancer. JAMA 309: 1493-1501, 2013.

34. Tang HC and Chen YC: Insight into molecular dynamics simulation of BRAF(V600E) and potent novel inhibitors for malignant melanoma. Int J Nanomedicine 10: 3131-3146, 2015.

35. Eloy C, Santos J, Soares P and Sobrinho-Simões M: The preeminence of growth pattern and invasiveness and the limited influence of BRAF and RAS mutations in the occurrence of papillary thyroid carcinoma lymph node metastases. Virchows Arch 459: 265-276, 2011.

36. Cheng S, Serra S, Mercado M, Ezzat S and Asa SL: A high-throughput proteomic approach provides distinct signatures for thyroid cancer behavior. Clin Cancer Res 17: 2385-2394, 2011. 